\title{
Krein condition in probabilistic moment problems
}

\author{
JORDAN STOYANOV \\ Department of Statistics, University of Newcastle, Newcastle upon Tyne NE1 7RU, UK. \\ E-mail: jordan.stoyanov@ncl.ac.uk
}

In 1944 M.G. Krein proposed a condition throwing light on the moment problem for absolutely continuous probability distributions. This condition, implying non-uniqueness, is expressed in terms of a normalized logarithmic integral of the density and has different forms in the Hamburger moment problem (for distributions on the whole real line) and in the Stieltjes moment problem (for distributions on the positive real line). Other forms of the Krein condition, together with new conditions (smoothing and growth condition on the density) suggested by G.D. Lin and based on a work by H. Dym and H.P. McKean, led to a unique solution to the moment problem. We present new results, give new proofs of previously known results and discuss related topics.

Keywords: determinate probability distributions; Hamburger moment problem; indeterminate probability distributions; Krein condition; Lin condition; moment sequence; powers of distributions; Stieltjes moment problem

\section{Introduction}

Suppose $F$ is a distribution function (d.f.) on the real line such that the $k$ th-order moments $\alpha^{(k)}=\int x^{k} \mathrm{~d} F(x), k=1,2, \ldots$, are all finite. Thus $F$ generates in a unique way the moment sequence $\left\{\alpha^{(k)}, k=1,2, \ldots\right\}$. We are interested in the inverse question: When does the moment sequence $\left\{\alpha^{(k)}\right\}$ uniquely determine $F$ ? Or, if $F$ and $G$ are d.f.s and $\int x^{k} \mathrm{~d} F=\int x^{k} \mathrm{~d} G$ for all $k=1,2, \ldots$, does this imply that $F=G$ ? If the answer is 'yes', we say that the moment problem has a unique solution ( $F$ is M-determinate). Otherwise, this problem has a non-unique solution ( $F$ is $M$-indeterminate), where ' $\mathrm{M}$ ' stands for 'moment'.

In this paper we deal with the moment problem in its probabilistic setting. All conditions and statements are expressed in terms of random variables (r.v.s) defined on an underlying probability space $(\Omega, \mathscr{T}, P)$ and their d.f.s and densities. For other aspects of the moment problem the reader is referred to the classical books by Shohat and Tamarkin (1943) and Akhiezer (1965), or to more recent books by Krein and Nudelman (1977), Landau (1987) and Koosis (1988).

One of the main and most frequently used tools in the moment problem is the Carleman criterion (see, for example Feller 1971; or Lukacs 1970): if $F$ is a d.f. on $(-\infty, \infty)$ with all moments finite, then the condition $\sum_{k=1}^{\infty}\left(\alpha^{(2 k)}\right)^{-1 / 2 k}=\infty$ implies that $F$ is unique; if $(0, \infty)$ is the support of $F$, then $\sum_{k=1}^{\infty}\left(\alpha^{(k)}\right)^{-1 / 2 k}=\infty$ guarantees the uniqueness of $F$. 
Let us focus now our attention on absolutely continuous distributions on $(-\infty, \infty)$ or on $(0, \infty)$ and describe the solution to the moment problem in terms of the so-called Krein condition. This condition involves the 'value' of the logarithmic normalized integral $K:=\int\left[(-\ln f(\cdot)) /\left(1+x^{2}\right)\right] \mathrm{d} x$, where $f$ is the density of $F$, assuming $f(x)>0$ for all $x$. The integral is taken over the support of $F$. The argument of $f(\cdot)$ is $x$ if the support is $(-\infty, \infty)$, see (1) and (2) below; this is the Hamburger moment problem. The density $f(\cdot)$ has an argument $x^{2}$ if the support is $(0, \infty)$, see (4) and (5) below; this is the Stieltjes moment problem. In general, there are two possibilities for $K$ : either $K$ is a finite number or $K$ is $+\infty$ (see Berg 1995; and Lin 1997). The solution of the moment problem depends only on whether $K=+\infty$ or $K<+\infty$.

The Krein condition appeared a long time ago (see Krein 1944). Curiously, it came when solving an extrapolation problem proposed by A.N. Kolmogorov. Krein provided a condition (see (1) below) which is sufficient for a distribution to be M-indeterminate. Hence the Krein result is opposite to conclusions based on the Carleman criterion. Note that the Carleman condition is not necessary for uniqueness, while the Krein condition is not necessary for non-uniqueness in the moment problem (see Section 11 in Stoyanov 1997).

Note that the Krein condition is intensively discussed by Akhiezer (1965) and briefly mentioned by Prohorov and Rozanov (1969). Chapter 5 in Koosis (1988) is devoted to the logarithmic normalized integral and the associated moment problems. The relation between the Carleman condition and the Krein condition is analysed in Dym and McKean (1976). This analysis culminated later in the discovery of conditions under which a distribution is Mdeterminate (see Lin 1997). It is regrettable that for some time the Krein condition (2) was misinterpreted. This began when Leipnik (1981) stated that (2) is a necessary and sufficient condition for uniqueness. This error was later repeated in other works such as Devroye (1986), Crow and Shimizu (1988). Slud (1993) made essential progress in the field, providing precise conditions for uniqueness and for non-uniquness in both the Hamburger and the Stieltjes moment problems. The next contributions come from works by Berg (1995; 1998a; 1998b), Lin (1997), Simon (1998) and Pedersen (1998). Illustrations and applications of the Krein condition can also be found in Stoyanov (1997; 1999) and Lin and Huang (1997).

\subsection{Hamburger moment problem}

Suppose the support of $F$ is $(-\infty, \infty)$, the density $f(x)>0$ for all $x \in(-\infty, \infty)$ and that all moments $\alpha^{(k)}=\int_{-\infty}^{\infty} x^{k} f(x) \mathrm{d} x, k=1,2, \ldots$, are finite.

Theorem 1. Suppose the following Krein condition is satisfied:

$$
\int_{-\infty}^{\infty} \frac{-\ln f(x)}{1+x^{2}} \mathrm{~d} x<\infty
$$

Then the d.f. F is M-indeterminate, i.e. the moment problem has a non-unique solution.

This statement can be derived from more general results of Krein (1944). The proof and several comments are available in Akhiezer (1965). It is remarkable to see different proofs 
in Slud (1993), Berg (1995) and Lin (1997) based on different ideas. Theorem 2 below is given in a form close to that in Lin (1997).

Theorem 2. Suppose the following Krein condition is satisfied:

$$
\int_{-\infty}^{\infty} \frac{-\ln f(x)}{1+x^{2}} \mathrm{~d} x=\infty
$$

Additionally, let the following Lin condition be satisfied: $f$ is symmetric, differentiable and, for some $x_{0}>0$ and $x \geqslant x_{0}$, we have

$$
\frac{-x f^{\prime}(x)}{f(x)} \nearrow \infty \text { as } x \rightarrow \infty .
$$

Then the d.f. $F$ is M-determinate, i.e. the moment problem has a unique solution.

\subsection{Stieltjes moment problem}

Now the support of the d.f. $F$ is the real half-line $(0, \infty)$. Suppose that $f(x)>0$, for all $x \in(0, \infty)$, and that all moments $\alpha^{(k)}=\int_{0}^{\infty} x^{k} f(x) \mathrm{d} x, k=1,2, \ldots$ are finite. Let us formulate two crucial results. Theorem 3 is due to Slud (1993) and its proof is based on an appropriate symmetrization procedure. Theorem 4 is given almost as in Lin (1997).

Theorem 3. Suppose the following Krein condition is satisfied:

$$
\int_{0}^{\infty} \frac{-\ln f\left(x^{2}\right)}{1+x^{2}} \mathrm{~d} x<\infty
$$

Then the d.f. F is M-indeterminate, i.e. the moment problem has a non-unique solution.

Theorem 4. Suppose the following Krein condition is satisfied:

$$
\int_{0}^{\infty} \frac{-\ln f\left(x^{2}\right)}{1+x^{2}} \mathrm{~d} x=\infty
$$

Additionally, let the Lin condition be satisfied: $f$ is differentiable and, for some $x_{0}>0$ and $x \geqslant x_{0}$, we have

$$
\frac{-x f^{\prime}(x)}{f(x)} \nearrow \infty \text { as } x \rightarrow \infty .
$$

Then the d.f. $F$ is M-determinate, i.e. the moment problem has a unique solution.

Remark 1. As noted by Lin (1997), the conditions in Theorems 2 and 4, together with arguments from Dym and McKean (1976), imply that the Carleman condition is satisfied, thus concluding the uniqueness of a distribution. In general, in order to check the Carleman condition we need to know all moments and show divergence of an infinite series. In Theorems 1-4 we operate only with the densities, which in many cases (see, for example, the 
next section) is much easier, with no need to calculate moments. All these conditions Carleman, Krein, Lin, etc. - appropriately chosen, are powerful tools in the moment problem.

\section{New results and new proofs of known results}

We now consider some popular probability distributions and treat the moment problem for them. We will see how easy is to derive new results or to obtain previously known results. If, however, the result is 'old', then its proof based on the Krein condition is new.

For our conclusions we need the following integrals (see, for example, Gradshteyn and Ryzhik 1980):

$$
\begin{aligned}
& \int_{0}^{\infty} \frac{x^{b}}{1+x^{2}} \mathrm{~d} x=\frac{\pi}{2 \cos (b \pi / 2)} \text { for } b \in(-1,1), \\
& \int_{0}^{\infty} \frac{\ln x}{a^{2}+x^{2}} \mathrm{~d} x=\frac{\pi}{2 a} \ln a \quad \text { for } a>0, \\
& \int_{0}^{\infty} \frac{(\ln x)^{2}}{1+x^{2}} \mathrm{~d} x=\frac{\pi^{3}}{8} .
\end{aligned}
$$

\subsection{Lognormal distribution}

Let $X$ be a positive r.v. with support $(0, \infty)$. We say that $X$ has a (standard) lognormal distribution with parameters $(0,1)$ and write $X \sim L(0,1)$ or $\mathscr{B}(X)=L(0,1)$, if its density is

$$
f(x)= \begin{cases}\frac{1}{x \sqrt{2 \pi}} \exp \left[-\frac{1}{2}(\ln x)^{2}\right], & \text { if } x>0, \\ 0, & \text { if } x \leqslant 0 .\end{cases}
$$

We easily find that $\alpha^{(k)}=\mathrm{E}\left[X^{k}\right]=\exp \left(k^{2} / 2\right), k=1,2, \ldots$, so $X$ has finite moments of all orders. The support of $\mathscr{L}(X)$ is $(0, \infty)$ and we are looking for the solution to the Stieltjes moment problem. The Carleman condition $\sum_{k=1}^{\infty}\left(\alpha^{(k)}\right)^{-1 / 2 k}=\infty$ is not satisfied in this case. However, even if satisfied, this condition is only sufficient without being necessary for the uniqueness of the moment problem (see Example 11.10 in Stoyanov 1997). Another possibility is to consider the series $\sum_{k=0}^{\infty} t^{k} \mathrm{e}^{k^{2} / 2} / k$ !, which is a 'candidate' for the moment generating function (m.g.f.) of $X$. However, this series diverges for any $t>0$, which means that $X$ does not have an m.g.f. (despite the existence of all moments!). In general (see Lukacs 1970), the existence of the m.g.f. implies the uniqueness of the distribution. No conclusion can be derived based on the non-existence of the m.g.f. Let us illustrate how easy it is to apply the Krein condition.

Proposition 1. Let $X$ be an r.v. with lognormal distribution $L(0,1)$. Then, for all real numbers $r$, except $r=0$, the distribution $\mathscr{L}\left(X^{r}\right)$ is M-indeterminate, i.e. the moment problem for $X^{r}$ has a non-unique solution. 
Proof. If $r=0, X^{0}=1$ a.s. is a degenerate r.v., its distribution unique. So we turn to $r \neq 0$.

Let $r>0$ and let $f_{r}$ denote the density function of the 'new' r.v. $X^{r}$. The support of $f_{r}$ is again $(0, \infty)$. Clearly, like $X$, in view of the Lyapunov inequality, $X^{r}$ also has finite moments of all orders for any $r>0$. We can find directly the density $f_{r}$ of $X^{r}$ or use the fact (Pakes and Khattree 1992), that the $r$ th power of $X, X^{r}$, also has a lognormal distribution denoted in this case by $L\left(0, r^{2}\right)$ and defined by the density

$$
f_{r}(x)= \begin{cases}\frac{1}{x r \sqrt{2 \pi}} \exp \left[-\frac{1}{2 r^{2}}(\ln x)^{2}\right], & \text { if } x>0, \\ 0, & \text { if } x \leqslant 0 .\end{cases}
$$

Writing first $f_{r}\left(x^{2}\right)$ for $f_{r}(x)$ given by (9) and using the integrals (7), we easily see that

$$
K_{r}:=\int_{0}^{\infty} \frac{-\ln f_{r}\left(x^{2}\right)}{1+x^{2}} \mathrm{~d} x<\infty \quad \text { for all } r>0 .
$$

Therefore the Krein condition (4) is satisfied and Theorem 3 says that the distribution $\mathscr{L}\left(X^{r}\right)$ is M-indeterminate for any $r>0$. This implies also that $\mathscr{B}\left(X^{-r}\right)$ is M-indeterminate for $r>0$ since $X=\mathrm{e}^{\xi}$ for an r.v. $\xi \sim N(0,1)$ and in view of the symmetry of $\xi$. Hence the distribution $\mathscr{B}\left(X^{r}\right)$ is M-indeterminate for any $r \neq 0$.

Remark 2. The case $r=1$ is special: $X^{1}=X$ is just the lognormally distributed r.v. $X$ whose density is given by (8). Proposition 1 yields that $L(0,1)=\mathscr{B}(X)$ is M-indeterminate, i.e. the moment problem for the standard lognormal distribution has a non-unique solution. In fact, this result, albeit in another form, was discovered by T. Stieltjes in 1894; details are given in Berg (1995). The term 'lognormal distribution' was introduced later. Since the appearance of the paper by Heyde (1963) this distribution has been among the most frequently cited as being not determined uniquely by its moment sequence. Note that Heyde does not use the Krein condition; he follows a different but effective idea, describing explicitly an infinite family of non-lognormal distributions each having the same moments as those of the lognormal distribution. Details can be seen in the original paper by Heyde (1963), but also in other sources such as Pakes and Khattree (1992), Berg (1995) or Stoyanov (1997).

\subsection{Powers of the normal distribution}

Suppose $X$ is an r.v. with a normal distribution $N\left(\mu, \sigma^{2}\right), \mu \in \mathbb{R}^{1}, \sigma^{2}>0$. All the moments of $X$ are finite, the m.g.f. $\mathrm{E}[\exp (t X)]=\exp \left(\mu t+\frac{1}{2} t^{2} \sigma^{2}\right)$ exists for all real $t$, so the normal distribution is M-determinate. The same conclusion can be derived for the distribution $\mathscr{C}\left(X^{2}\right)$, and the next question is about the powers $X^{m}$ for positive integers $m \geqslant 3$, or $|X|^{r}$ for real $r>0$. (If $r=0$, the distribution of the degenerate r.v. $|X|^{0}=1$ is unique, which is not so interesting.)

Proposition 2. Let $X$ be a normally distributed r.v. Then the following statements hold:

(a) The distribution $\mathscr{L}\left(X^{2 n+1}\right)$ is M-indeterminate for any $n=1,2, \ldots$. 
(b) The distribution $\mathscr{B}\left(|X|^{r}\right)$ is M-determinate for all $r \in(0,4]$.

(c) The distribution $\mathscr{L}\left(|X|^{r}\right)$ is M-indeterminate for all $r>4$.

Proof. Claims (a), (b) and (c) are among the main results in Berg (1988). Berg's approach when proving them is to write explicitly infinite families of distributions with the same moments in cases of indeterminacy, or to use the Carleman criterion in the case of determinacy. The Krein condition is not used. Our approach here is to use the Krein-Lin technique and offer easier proofs of the same statements.

Clearly claims (a), (b) and (c) do not depend on the specific values of the parameters of the normal distribution. Thus, for simplicity, we assume that $X \sim N\left(0, \frac{1}{2}\right)$, so the density $f$ of $X$ is $f(x)=(1 / \sqrt{\pi}) \exp \left(-x^{2}\right), x \in(-\infty, \infty)$.

For our next steps we need the explicit expressions for the densities $f_{2 n+1}$ of $X^{2 n+1}$ and $g_{r}$ of $|X|^{r}$. Standard arguments lead to the following formulae:

$$
\begin{aligned}
& f_{2 n+1}(x)=\frac{1}{(2 n+1) \sqrt{\pi}}|x|^{-2 n /(2 n-1)} \exp \left[-|x|^{2 /(2 n+1)}\right], \quad x \in(-\infty, \infty), n=1,2, \ldots, \\
& g_{r}(x)= \begin{cases}\frac{2}{r \sqrt{\pi}} x^{1 / r-1} \exp \left[-x^{2 / r}\right], & x>0, r>0 ; \\
0, & x \leqslant 0 .\end{cases}
\end{aligned}
$$

There is an obvious difference: $X^{2 n+1}$ is distributed on $(-\infty, \infty)$, which is a Hamburger case, while $|X|^{r}$ is distributed on $(0, \infty)$, which is a Stieltjes case. When finding the quantity

$$
K_{2 n+1}:=\int_{-\infty}^{\infty} \frac{-\ln f_{2 n+1}(x)}{1+x^{2}} \mathrm{~d} x
$$

we use (10) to obtain $K_{2 n+1}=C+I_{1}+I_{2}$, where

$$
C=\text { const. }, \quad I_{1}=\frac{2 n}{2 n-1} \int_{-\infty}^{\infty} \frac{-\ln |x|}{1+x^{2}} \mathrm{~d} x, \quad I_{2}=\int_{-\infty}^{\infty} \frac{|x|^{2 /(2 n+1)}}{1+x^{2}} \mathrm{~d} x .
$$

We have $I_{1}=(4 n /(2 n-1)) \int_{0}^{\infty}\left[(\ln x) /\left(1+x^{2}\right)\right] \mathrm{d} x=0$ in view of the second integral in (7). Since $2 /(2 n+1)<1$ for any $n=1,2, \ldots$, the first integral in (7) implies that $I_{2}<\infty$. Hence $K_{2 n+1}<\infty$, the Krein condition (1) is satisfied and, according to Theorem 1, the distribution $\mathscr{B}\left(X^{2 n+1}\right)$ is M-indeterminate for any $n=1,2, \ldots$

Now we turn to the quantity

$$
K_{r}^{*}:=\int_{0}^{\infty} \frac{-\ln g_{r}\left(x^{2}\right)}{1+x^{2}} \mathrm{~d} x .
$$

For $g_{r}$ from (11) we write first $g_{r}\left(x^{2}\right)$ and take $-\ln g_{r}\left(x^{2}\right)$, thus obtaining $K_{r}^{*}=C_{2}+J_{1}+$ $J_{2}$, where

$$
C_{2}=\text { const. }, \quad J_{1}=-\left(\frac{2}{r}-2\right) \int_{0}^{\infty} \frac{\ln x}{1+x^{2}} \mathrm{~d} x, \quad J_{2}=\int_{0}^{\infty} \frac{x^{4 / r}}{1+x^{2}} \mathrm{~d} x .
$$


Again, the second integral in (7) implies that $J_{1}=0$ for all $r>0$. Thus the dominating term in $K_{r}^{*}$ is $J_{2}$. Since $r>0$, in view of the first integral in (7) we obtain that

$$
J_{2}<\infty \Leftrightarrow 4 / r<1 \Leftrightarrow r>4 .
$$

Hence $K_{r}^{*}<\infty$ for these values of $r$, the Krein condition (4) is satisfied and Theorem 3 tells us that the distribution $\mathscr{B}\left(|X|^{r}\right)$ is M-indeterminate for any real $r>4$.

Moreover, for any $r \in(0,4], J_{2}=\infty$, which is exactly the Krein condition (5). Hence in this case we have to check if the Lin condition (6) holds. We find

$$
\frac{-x g_{r}^{\prime}(x)}{g_{r}(x)}=-\left(\frac{2}{r}-2\right)+\frac{4}{r} x^{4 / r} \nearrow \infty \quad \text { as } x \rightarrow \infty .
$$

Thus condition (6) is satisfied even for any $r>0$, not only for $r \in(0,4]$. Therefore, according to Theorem 4, the distribution $\mathscr{b}\left(|X|^{r}\right)$ is M-determinate for any $r \in(0,4]$.

\subsection{Powers of the inverse Gaussian distribution}

We say that the r.v. $X$ taking positive values has an inverse Gaussian distribution with parameters $\mu>0, \lambda>0, X \sim I G(\mu, \lambda)$, if $X$ is absolutely continuous with density

$$
f(x)= \begin{cases}\left(\frac{\lambda}{2 \pi x^{3}}\right)^{1 / 2} \exp \left[-\frac{\lambda}{2 \mu^{2}} \frac{(x-\mu)^{2}}{x}\right], & \text { if } x>0, \\ 0, & \text { if } x \leqslant 0 .\end{cases}
$$

First we can check that $X$ has finite moments $\alpha^{k}=\mathrm{E}\left[X^{k}\right]$, for all $k=1,2, \ldots$ Moreover, the m.g.f. $M_{X}(t)=\mathrm{E}[\exp (t X)]$ is explicitly known (see, for example, Seshadri 1993):

$$
M_{X}(t)=\exp \left\{(\lambda / \mu)\left[1-\left(1-2 \mu^{2} t / \lambda\right)^{1 / 2}\right]\right\}
$$

Obviously, $M_{X}(t)$ is well defined for all $t<\lambda /\left(2 \mu^{2}\right)$, which means that $M_{X}$ exists in a proper neighbourhood of $t=0$. Then a general theorem in Lukacs (1970) yields that the distribution $\mathscr{L}(X)$, i.e. the inverse Gaussian distribution, is M-determinate. So for what values of $r$ the distribution $\mathscr{L}\left(X^{r}\right)$ of $X^{r}$ is M-indeterminate?

Proposition 3. Let $X \sim I G(\mu, \lambda)$ and let $r$ be a real number. The following statements hold:

(a) If $r<-2$ or $r>2$, then the distribution $\mathscr{B}\left(X^{r}\right)$ is M-indeterminate.

(b) If $-2 \leqslant r \leqslant 2$, then the distribution $\mathscr{B}\left(X^{r}\right)$ is M-determinate.

Sketch of proof. The natural idea is to find the density of $X^{r}$ and check which form of the Krein condition is satisfied, reaching our conclusion directly or after testing the Lin condition (6). Details as well as some related topics are given in Stoyanov (1999). 


\section{Functions preserving or destroying the determinacy}

It turns out that for an r.v. $X$ having some of the popular distributions, $\mathscr{B}(X)$ and $\mathscr{B}\left(X^{2}\right)$ are M-determinate while $\mathscr{B}\left(X^{3}\right)$ is M-indeterminate and the value $r=3$ is the minimal integer positive power such that the distribution $\mathscr{L}\left(X^{r}\right)$ or $\mathscr{B}\left(|X|^{r}\right)$ becomes M-indeterminate. It was of interest, for a few cases, to raise the curious question of the mysterious role of the number 3 as a power of $X$ when we 'lose' the uniqueness in the moment problem.

Let us recall some definitions. We say that $X$ has the gamma distribution with parameters $a>0, b>0$ and write $X \sim \gamma(a, b)$ if the density of $X$ is

$$
f(x)= \begin{cases}{\left[b^{a} / \Gamma(a)\right] x^{a-1} \exp [-b x],} & x>0, \\ 0, & x \leqslant 0 .\end{cases}
$$

If $X \sim \gamma(a, b)$ and $a=1$, then $X$ is exponentially distributed, $X \sim \operatorname{Exp}(b)$. And $X$ has Laplace distribution (double-exponential) if the density of $X$ is $(b / 2) \exp (-b|x|), x \in$ $(-\infty, \infty)$. Finally, we say that the r.v. $X$ has a logistic distribution if the density of $X$ is $f(x)=\mathrm{e}^{-x} /\left(1+\mathrm{e}^{-x}\right)^{2}, x \in(-\infty, \infty)$.

In the following statements, $r=3$ is the minimal integer positive value of the power $r$ when the distribution $\mathscr{B}\left(X^{r}\right)$ or $\mathscr{B}\left(|X|^{r}\right)$ becomes M-indeterminate.

(a) If $X$ is normally distributed, then $\mathscr{L}\left(X^{3}\right)$ is M-indeterminate.

(b) If $X$ is exponentially distributed, then $\mathscr{L}\left(X^{3}\right)$ is M-indeterminate.

(c) If $X$ has the Laplace (double-exponential) distribution, then $\mathscr{B}\left(|X|^{3}\right)$ is $\mathrm{M}$ indeterminate.

(d) If $X$ has the gamma distribution, then $\mathscr{B}\left(X^{3}\right)$ is M-indeterminate.

(e) If $X$ has the logistic distribution, then $\mathscr{L}\left(X^{3}\right)$ is M-indeterminate.

(f) If $X$ has inverse Gaussian distribution, then $\mathscr{B}\left(X^{3}\right)$ is M-indeterminate.

Claim (a), mentioned by Prohorov and Rozanov (1969), is discussed by Berg (1988). Claims (b), (c) and (d) and their modifications are considered in Targhetta (1990), Pakes and Khattree (1992) and Lin and Huang (1997); see also Example 11.5 in Stoyanov (1997). Claim (e) is one of the results in Lin and Huang (1997), while claim (f) follows from Proposition 3 above; see also Stoyanov (1999). Cases (a)-(d), as presented in the works cited, do not rely on the Krein-Lin techniques.

The question above was about the role of the power 3. That this is a coincidence follows from claim (c) of Proposition 2 above, implying that $r=5$ (not 3 ) is the minimal positive integer value of $r$ when $\mathscr{L}\left(|X|^{r}\right)$ becomes M-indeterminate.

Pakes and Khattree (1992) and Lin and Huang (1997) contain several related results for the generalized gamma distributions. The deep analysis in Slud (1993) of the determinacy of the distribution of polynomials in normal r.v.s motivates us to raise such a question for polynomials in r.v.s having another distribution, such as exponential or inverse Gaussian.

Look also at the logarithmic function destroying the uniqueness of the normal distribution. The reader may find it interesting to check whether or not the log-exponential distribution is M-determinate. However, if we take an r.v. $X$ with beta distribution on $(0,1)$ and define the 'new' r.v. $Y:=-\ln X$, with the $\log$-beta distribution, we easily obtain that 
the distribution $\mathscr{L}(Y)$, having $(0, \infty)$ as its support, is M-determinate. So, in this case the logarithmic function preserves the determinacy. Note finally that this function destroys the uniqueness of the Poisson distribution: the log-Poisson distribution is M-indeterminate (see Example 11.7 in Stoyanov 1997).

Our interest is not confined to powers and the logarithmic function. It is interesting to take an arbitrary functional transformation $Y:=h(X)$, where $X$ is a given r.v. and $h$ is an appropriate real-valued function. Assume $X$ and $Y$ have all moments finite. Each of the distributions $\mathscr{L}(X)$ and $\mathscr{B}(Y)$ is either M-determinate or M-indeterminate. Avoiding a few trivial cases, we want to characterize pairs $X, h$ such that the determinacy property is preserved, i.e. both $\mathscr{L}(X)$ and $\mathscr{L}(Y)$ are M-determinate or M-indeterminate. Cases when the determinacy property is changed to that of indeterminacy are also of interest. This topic is currently under study.

\section{Comments on related topics}

Comment 1. There are discrete distributions which are M-indeterminate; examples of this kind can be seen in Section 11 of Stoyanov (1997) and other families were given recently by Berg (1998a, 1998b). The question is to find an analogue of each of the forms of the Krein condition for purely discrete distributions and exploit them for the moment problem. Perhaps the paper by Pedersen (1998), treating the Hamburger moment problem, is the only contribution in this direction.

Comment 2. Many distributions, including those in Section 2, can be treated in a unified way. To achieve this we assume that the r.v. $X$ is absolutely continuous with distribution belonging to the exponential family: the density of $X$ has the form $f(x)=A u(x) \exp [-v(x)]$, where $u$ and $v$ are appropriate positive functions (on the support of $f$ ) and $A$ is a normalizing constant. Assuming $X$ has all moments finite, we are interested in the corresponding Hamburger or Stieltjes moment problem. In this case Theorems 1-4 can be reformulated in such a way that the Krein and Lin conditions be expressed only in terms of the two functions $u$ and $v$. Then as a consequence we can obtain many known results.

Comment 3. The works by Berg and Christensen (1981) and Berg (1998b) show the nontrivial result that if the d.f. $F$ is M-indeterminate, then there are 'many' discrete as well as 'many' absolutely continuous distributions having the same moments of all orders ('many' means infinitely many!). Leipnik (1981) found explicitly discrete distributions having all moments finite and the same as those of the (absolutely continuous!) lognormal distribution; see also the comments by Pakes and Khattree (1992) and Berg (1998b). So, how to find discrete distributions (M-indeterminate!) having the same moments as those of the cube of the normal distribution, the cube of the exponential distribution, etc.?

Comment 4. The Krein-Lin techniques can be successfully applied when solving the identifiability problem for mixtures of distributions in the spirit of the recent paper by Sapatinas (1995). 


\section{Acknowledgements}

This paper was finished in August 1998 when I was still visiting the Universidade Federal do Rio de Janeiro (Brazil) as CNPq appointee, 1997/1998. Partial support was received from Ministry of Education and Science (Bulgaria) grant MM-705/97-BG G.

Professors C. Berg, G.D. Lin and A. Pakes expressed kindly their opinion on previous versions of the paper. I am grateful to them for their interest and attention and for their constructive suggestions. My thanks are addressed to the anonymous referees and to the Associate Editor for their comments, which were essential in preparing this final version of the paper.

This paper is dedicated to Professor Chris Heyde on the occasion of his 60th birthday.

\section{References}

Akhiezer, N.I. (1965) The Classical Moment Problem. New York: Hafner.

Berg, C. (1988) The cube of the normal distribution is indeterminate. Ann Probab., 16, 910-913.

Berg, C. (1995) Indeterminate moment problems and the theory of entire functions. J. Comput. Appl. Math., 65, 27-55.

Berg, C. (1998a) On some indeterminate moment problems for measures on a geometric progression. J. Comput. Appl. Math., 99, 67-75.

Berg, C. (1998b) From discrete to absolutely continuous solutions of indeterminate moment problems. Preprint No. 11, September 1998, University of Copenhagen.

Berg, C. and Christensen, J.P.R. (1981) Density questions in the classical theory of moments. Ann. Inst. Fourier, 31, 99-114.

Crow, E.L. and Shimizu, K. (1988) Lognormal Distributions: Theory and Applications. New York: Marcel Dekker.

Devroye, L. (1986) Non-Uniform Random Variate Generation. New York: Springer-Verlag.

Dym, H. and McKean, H.P. (1976) Gaussian Processes, Function Theory and the Inverse Spectral Problem. New York: Academic Press.

Feller, W. (1971) An Introduction to Probability Theory and Its Applications, Vol. 2, 2nd edn. New York: Wiley.

Gradshteyn, I.S. and Ryzhik, I.M. (1980) Tables of Integrals, Series and Products. New York: Academic Press.

Heyde, C.C. (1963) On a property of the lognormal distribution. J. Roy. Statist. Soc. Ser. B, 29, 392393.

Koosis, P. (1988) The Logarithmic Integral, Vol. 1, Cambridge: Cambridge University Press.

Krein, M.G. (1944) On one extrapolation problem of A.N. Kolmogorov. Dokl. Akad. Nauk SSSR, 46(8), 339-342 (in Russian).

Krein, M.G. and Nudelman, A.A. (1977). The Markov Moment Problem and Extremal Problems. Providence, RI: American Mathematical Society.

Landau, H.J. (ed.) (1987) Moments in Mathematics, Proc. Sympos. Appl. Math. 37. Providence, RI: American Mathematical Society.

Leipnik, R. (1981) The lognormal distribution and strong nonuniqueness of the moment problem. Theory Probab. Appl., 26, 850-852.

Lin, G.D. (1997) On the moment problem. Statist. Probab. Lett., 35, 85-90. 
Lin, G.D. and Huang, J.S. (1997) The cube of the logistic distribution is indeterminate. Austral. $J$. Statist., 39, 247-252.

Lukacs, E. (1970) Characteristic Functions, 2nd edn. London: Griffin.

Pakes, A.G. and Khattree, R. (1992) Length-biasing, characterizations of laws and the moment problem. Austral. J. Statist., 34, 307-322.

Pedersen, H.L. (1998) On Krein's theorem for indeterminacy of the classical moment problem. $J$. Approx. Theory, 95, 90-100.

Prohorov, Yu.V. and Rozanov, Yu.A. (1969) Probability Theory. Berlin: Springer-Verlag.

Sapatinas, Th. (1995) Identifiability of mixtures of power-series distributions and related characterizations. Ann. Inst. Statist. Math., 47, 447-459.

Seshadri, V. (1993) The Inverse Gaussian Distribution. Oxford: Clarendon Press.

Shohat, J.A. and Tamarkin, J.D. (1943) The Problem of Moments. Providence, RI: American Mathematical Society.

Simon, B. (1998) The classical moment problem as a self-adjoint finite difference operator. $A d v$. Math., 137, 82-203.

Slud, E.V. (1993) The moment problem for polynomial forms of normal random variables. Ann. Probab., 21, 2200-2214.

Stieltjes, T.J. (1894) Recherches sur les fractions continués. Ann. Fac. Sci. Toulouse, 8, 1-122.

Stoyanov, J. (1997) Counterexamples in Probability, 2nd edn. Chichester: Wiley.

Stoyanov, J. (1999) Inverse Gaussian distribution and the moment problem. J. Appl. Statist. Sci., 9, 6171.

Targhetta, M.L. (1990) On a family of indeterminate distributions. J. Math. Anal. Appl., 147, 477479 .

Received October 1998 and revised September 1999 